\title{
Estimando o retorno à educação do Brasil considerando a legislação educacional brasileira como um instrumento
}

\author{
WLADIMIR MACHADO TEIXEIRA \\ NAÉRCIO AQUINO MENEZES-FILHO*
}

\begin{abstract}
Estimating the return to education the Brazilian legislation of education as an instrument considering. This paper aims to estimate the impact of education on wages in Brazil. GDP, Population, and the number of schools in the state and year when the individual was born as instruments for his education level were used. In this context, the paper consider other instrument, the Brazilian education Law 5692 of 1971. The results show that the Law 5692 of 1971 and the number of schools in the individual's year of birth bears a positive relationship with his education, and the returns to education decrease quite substantially when the method of instrumental variables is used.
\end{abstract}

Keywords: Mincer; instrumental variables; education.

JEL Classification: J30; J31; I21.

\section{INTRODUÇÃO}

O objetivo deste artigo consiste em estimar o efeito da educação sobre os salários no Brasil, e, considera o problema de endogeneidade da variável educação ao utilizar o número de escolas no Estado e no ano da data de nascimento do indivíduo como variável instrumental. Além do número de escolas, o trabalho abordará os efeitos de outros instrumentos sobre a probabilidade de escolarização, tais como: população, leis que afetem a probabilidade de escolarização, e PIB.

O trabalho admite o problema de endogeneidade da educação na equação de salários. Desta forma, o artigo mencionará a importância de se adotar a relação

\footnotetext{
* Universidade Federal do Mato Grosso do Sul, e-mail wladimirteixeira@zipmail.com.br; Insper e Universidade de São Paulo, e-mail NaercioAMF@isp.edu.br. Submetido: 30/março/2010; Aprovado: 19/agosto/2011.
} 
log-linear de Mincer (1974). Além disso, discutir-se-á o método de variáveis instrumentais ao considerar o problema de endogeneidade da variável educação.

Serão estimados modelos de Mínimos Quadrados Ordinários e de Variáveis Instrumentais que mostram o papel da educação ao longo das gerações como função: dos estados brasileiros, das participações dos Produtos Internos Brutos de cada estado no Brasil, da Lei 5692 de 1971, da população de cada estado ao longo dos anos, e do impacto do ciclo de vida através das variáveis idade e idade ao quadrado. Nesta análise, serão agregadas várias Pesquisas Nacionais por Amostras Domiciliares de 1997 a 2007 e serão incluídas as dummies de ano e dummies de Estado. O trabalho mostrará a equação de rendimentos e o retorno à educação quando são utilizadas as escolas no estado e no ano de nascimento dos indivíduos como instrumentos para a variável educação.

Então, além do número de escolas, população, e, PIB, pode-se dizer que existiram leis que afetaram na decisão educacional do aluno? Então, ao tentar resporder esta pergunta o trabalho avança em relação aos demais ao propõr instrumentos que atentem para a endogeneidade da educação, entre os instrumentos pode ser destacada a Lei 5692 de 1971.

\section{O RETORNO À EDUCAÇÃO E A UTILIZAÇÃO DO MÉTODO DE VARIÁVEIS INSTRUMENTAIS}

A discussão entre retornos à educação e mercado de trabalho pode ser destacada desde o debate entre Adam Smith e Stuart Mill. Para Adam Smith os salários eram determinados apenas pela oferta e demanda por trabalho, logo, não haveria preocupação com a desigualdade salarial, o mercado de trabalho funcionava bem e o desemprego era ocasionado por diferenças de características pessoais ou habilidades. Por outro lado, Mill enfatizava que os salários seriam determinados por motivos não pecuniários e a desigualdade tenderia a aumentar. Portanto, existia a ideia de salários de eficiência, pois um salário mais elevado era uma compensação do esforço anterior para os indivíduos desenvolverem habilidades em algumas ocupações ${ }^{1}$.

Um aspecto bastante discutido na literatura relacionada à equação de salários refere-se à situação em que o diferencial de salários teria como fonte as características não produtivas, tais como: sexo e raça, o que seria caracterizado como discriminação no mercado de trabalho. Além disso, diferenças de rendimentos poderiam ser explicadas por diferenças nas características observáveis dos trabalhadores como, por exemplo, educação e experiência. Assim, mesmo em grupos não homogêneos, aspectos não pecuniários seriam importantes para explicar o diferencial de salários².

\footnotetext{
${ }^{1}$ Ver Fernandes (2002).

${ }^{2}$ Ver Fernandes (2002), Menezes-Filho (2002), Corseuil et al. (2002).
} 
Desta maneira, vários estudos mostram que, em média, os salários dos homens são superiores ao das mulheres mesmo após o controle por diversas características observáveis. Uma possível interpretação é que se trata de uma discriminação no mercado de trabalho. Entretanto, existem outras possibilidades, entre elas a necessidade de engajamento no trabalho.

Como forma de lidar com o problema de endogeneidade da variável educação, boa parte da literatura internacional adota estimadores de variáveis instrumentais para obter os retornos à educação. Então, o pesquisador considera a existência de uma covariável observável que afeta a decisão de escolaridade, mas não é correlacionada com fatores de habilidade.

Pode-se dizer que o estimador de mínimos quadrados ordinários tem viés de habilidade relativo ao retorno marginal da educação. Se o estimador de MQO é viesado devido a uma habilidade não observada, pode-se utilizar um estimador de variáveis instrumentais.

Um dos trabalhos pioneiros na área de economia da educação foi o de Griliches (1977), o qual considerou que a barganha entre patrões e empregados deve estabelecer preços relativos proporcionais a diferentes produtividades marginais de diferentes tipos de trabalho. Desta forma, a habilidade pode ser incorporada ao modelo através da seguinte equação:

$$
Y=\alpha+\beta S+\gamma A+u
$$

Então, A é a medida de habilidade, que tem sido ignorada em vários processos de estimação. Assim tem-se:

$$
E b_{y s}=\beta+\gamma b_{A S}=\beta+\gamma \operatorname{Cov}(A S) / \operatorname{Var} S
$$

O coeficiente de $S$ relacionado à variável educação seria viesado para cima (relativo ao $\beta$ ) a partir dos seguintes pressupostos: i) A habilidade tem um efeito positivo e independente sobre os rendimentos $(\gamma>0)$ e afeta a escolaridade acumulada; ii) A relação entre excluir habilidade e incluir variáveis de escolaridade é positiva $\left(b_{\mathrm{AS}}>0\right)$.

Dada a importância da variável habilidade, o problema passou a ser encontrar uma medida de habilidade e incluí-la na equação. Foram aceitos testes de QI, AFQT (teste de aptidão militar americano), ou testes psicológicos relevantes para mensurar habilidade. Trabalhos recentes se concentram em estimar um percentual de viés de habilidade, baseado na estimação de $\gamma \mathrm{b}_{\mathrm{AS}} \beta$. O grande problema é que não há razão para esperar que o viés de habilidade seja constante entre diferentes amostras.

Sempre que $\gamma$ for uma constante, a magnitude do viés de habilidade não observável irá depender de $\beta$ e $\mathrm{b}_{\mathrm{AS}}$. A estimativa de $\beta$ irá diferir entre estudos, dependendo, por exemplo, da parametrização da equação. No trabalho de Mincer a estimação de b considera a experiência constante.

Logo, há o debate sobre a heterogeneidade dos retornos à educação. Ashenfelter et al. (1999) observam que a maioria dos trabalhos empíricos na área de economia da educação têm utilizado variáveis instrumentais para o tratamento da 
heterogeneidade não observável da variável educação. Desde que vários instrumentos afetam a decisão de escolaridade de vários subgrupos e de subpopulações, é possível ter-se retornos à educação diferentes, e não é surpresa que estimativas de retornos à educação variem entre estudos.

Além disto, à luz da teoria do capital humano desenvolvido por Becker (1964), Mincer (1974), e Griliches (1977), pode-se dizer que Light (2001) afirma que os estudantes acumulam experiência de trabalho durante a escola. Muitos jovens acumulam uma quantidade de experiência de trabalho antes de completar sua escolaridade, trabalhando nas férias, trabalhando durante o ano acadêmico, e trabalhando durante as interrupções de poucos meses a poucos anos.

Os dados da National Longitudinal Survey of Youth (NLSY) mostram que estudantes americanos do secundário acumulam em média mil horas de experiência de trabalho entre os 16 anos e o término do ensino de segundo grau, enquanto um graduado na faculdade obtém 5 mil horas de trabalho de experiência no tempo que leva estudando. Dessa forma, Light (2001) questiona: Qual a relação entre escolaridade e salários após a escola? Então, cabe distinguir o efeito causal dos salários após o término dos estudos (tempo gasto em escolaridade), e experiência de trabalho quando o indivíduo está estudando (tempo de trabalho gasto quando ainda está na escola).

Usando dados do National Longitudinal Survey of Youth (NLSY), Light (2001) estimou modelos com e sem controles em experiência de trabalho na escola. Os coeficientes obtidos de escolaridade são 25\%-44\% maiores (dependendo de como se controla o viés de habilidade).

Loury e Garman (1995) afirmam que o desempenho na faculdade e a seletividade têm efeito significante sobre os rendimentos e varia entre negros e brancos. Trabalhos que não incluem o desempenho na faculdade superestimam o efeito de seletividade da faculdade entre brancos e negros. Este trabalho utilizou uma amostra representativa para estimar os efeitos de anos de faculdade, seletividade, gestão e desempenho de rendimentos de homens que tiveram quatro anos de faculdade. Pontuação na faculdade, bem como a escolha desta, tem efeito elevado e significante sobre os rendimentos. O ganho de salários para estudantes negros que tiram uma nota pior no SAT é significativamente menor na graduação, só não sendo superado pelo ganho dos que falharam por não completar a faculdade.

Os estudos de Card (1995) examinam o retorno à educação associado com crescimento educacional advindo do indivíduo morar próximo a uma faculdade ou universidade. Card (1995) acha que a proximidade da faculdade pode ser utilizada como um instrumento para escolaridade na amostra de NLS de homens jovens e encontra que o resultado do estimador de variáveis instrumentais (IV) está substancialmente acima do correspondente estimador de MQO. Além de Card (1995), Kane e Rouse (1993) acreditam também que estimativas de retornos à escolaridade por variáveis instrumentais (IV) baseado na proximidade da faculdade excedem as correspondentes estimativas de MQO por 20-30\%, dependendo dos controles que são adicionados no modelo.

Harmon e Walker (1995) examinam o retorno à educação em uma grande 
amostra de famílias de homens britânicos. Eles usam como variável instrumental para escolaridade um par de variáveis dummy que mostram a mudança de atendimento escolar por idade na Inglaterra - de até 14 anos para 15 anos em 1947, e de até 15 anos para 16 anos em 1973. Estas dummies de coorte efetivamente distinguem entre homens nascidos depois de 1932, ou seja, nascidos entre 1933 e 1957, e aqueles nascidos antes de 1957. Então, a mudança da lei de 1947, serviu como contrafactual para analisar os resultados após a Segunda Guerra Mundial. Harmon e Walker analisam que não houve um crescimento de atendimento de educação para consecutivas coortes de homens, já outra corrente acreditava que a mudança da lei de 1947 e 1973 seria responsável pelo aumento do atendimento escolar.

Já o estudo de Ichino, Winter, e Ebmer (1998) utiliza coorte de nascimento como uma fonte de variação da escolaridade. Em particular, focam no rendimento e escolaridade de homens austríacos e alemães nascidos entre 1930 e 1935. Eles argumentam que a Segunda Guerra Mundial tem um efeito forte e particular sobre o atendimento educacional diretamente sujeito a hostilidades. Usando dados de 14 países, eles encontraram uma grande diferença em educação completada por crianças em 1930-1935 na coorte de países que foram mais fortemente afetados pela guerra. Então, concluem que hostilidades afetam negativamente o retorno à educação.

Vislumbrou-se outra possibilidade de atentar para o problema de endogeneidade da educação, conforme Ashenfelter et al. (1999). Segundo estes autores, fatores como escolaridade compulsória ou acessibilidade de escola são mais preferíveis para afetar a escolha de escolaridade do indivíduo. Então, os estimadores de variáveis instrumentais baseados sobre escolaridade compulsória ou proximidade escolar deverão obter um retorno de educação estimado acima da correspondente estimativa de MQO.

Angrist e Krueger (1991) utilizam o trimestre da data de nascimento individual (interagindo com anos de nascimento ou estado de nascimento em algumas especificações) como instrumento para escolaridade para homens nascidos entre 1930 e 1959. O objetivo do artigo era estimar o efeito da lei de escolaridade compulsória sobre os retornos à educação. Os referidos autores notam que pessoas nascidas no mesmo ano calendário típico começam a estudar no mesmo tempo. Assumindo que o trimestre de nascimento é independente de fatores de preferências e habilidades, a lei de escolaridade compulsória gera uma variação exógena na educação que pode ser usada como um estimador de variável instrumental.

Já Duflo (2001), apresenta o experimento de construção de escolas sobre o mercado de trabalho na Indonésia e encontra que o aumento no atendimento educacional ocasionou aumento dos rendimentos. Depois de controlar por região de nascimento e os efeitos de coorte de nascimento, interagiu-se variáveis dummies indicando a idade do indivíduo em 1974 e a intensidade do programa na região de nascimento. Tentou-se mensurar o efeito do número de escolas construídas entre 1973-1974 e entre 1978-1979 na Indonésia.

Os dados de Duflo (2001) originam-se da amostra da Indonésia (SUPAS) e focaram em homens nascidos entre 1950 e 1972. A data e a região de nascimento 
determinam conjuntamente uma exposição individual ao programa de construção de escolas. Combinando o efeito do programa sobre anos de educação e salários, tem-se o retorno à educação em torno de $6.8 \%$ e $10.6 \%$ por cento.

Conforme visto, apresentamos uma parte da literatura internacional sobre retornos à educação e a problemática em torno da endogeneidade, inclusive apontando soluções para o tema. Cabe agora mostrar a problemática do fato da variável educação ser endógena segundo a literatura nacional. Desta maneira, Langoni (1973) foi um dos pioneiros no estudo dos diferenciais de salários por escolaridade, encontrando diferenças salariais significativas entre trabalhadores com diferentes anos de estudo.

Depois de Langoni (1973), Senna (1976), utilizando dados do Ministério do Trabalho, encontra um retorno de $14 \%$ para um ano adicional de educação. Seu universo se restringe a homens na área urbana trabalhando no setor formal em 1970. O autor usa como variável de controle a experiência do trabalhador. Já Tannen (1991), mostra que o retorno para cada ano adicional de estudo é de $12,8 \%$ e $8,1 \%$ respectivamente, para o primeiro e o segundo ciclo do ensino básico, $15,7 \%$ para o segundo grau e $23,4 \%$ para o ensino superior. Um ano adicional do ensino superior apresenta maior impacto sobre os salários.

Como vimos na seção anterior, a relação entre educação e salário está sujeita ao problema de endogeneidade. É possível que certas características usualmente não investigadas estejam correlacionadas tanto ao nível educacional como ao salário, tal como quociente de inteligência, boa capacidade de relacionamento (inteligência emocional) etc.

Behrman e Birdsall (1983) tentam uma forma de identificar a relação entre salários e educação, e argumentam que a qualidade da educação adquirida pelo indivíduo pode estar correlacionada tanto ao salário como a quantidade de educação adquirida. Os autores conduzem uma análise empírica para o Brasil usando dados do Censo Demográfico de 1970. De fato, quando os autores incorporam uma proxy de qualidade ${ }^{3}$ para a educação na equação de salário, o retorno estimado para a educação cai consideravelmente em comparação com as estimativas de um modelo sem essa proxy.

Além do problema de identificação, Birdsall e Behrman (1983) chamam a atenção para um possível viés das estimativas de retornos à educação que aparece em dados agregados espacialmente. Os autores justificam que os fatores regionais podem estar correlacionados tanto ao desempenho educacional como aos salários, bem como a diferenças no custo de vida entre regiões.

Ramos (1991), Leal e Werlang (1991), e Barros e Ramos (1994) fazem um esforço semelhante ao de Tannen (1991) para identificar os retornos à educação no Brasil. Além de considerar que o efeito da educação pode ser diferenciado de acordo com a etapa do ciclo educacional, também se preocupam em identificar como a evolução no tempo do retorno associado a cada etapa do ciclo educacional.

\footnotetext{
${ }^{3}$ Educação média dos professores do município em que o indivíduo obteve escolaridade.
} 
No que se refere ao impacto da variável educação, Ramos (1991) considera o período investigado de 1976 a 1985 e incorpora o setor de atividade e a posição na ocupação por controles. Leal e Werlang (1991) consideram o período entre 1976 a 1989, e além de educação, consideram a experiência como variável explicativa. Já Barros e Ramos (1994) consideram, além da experiência, idade e região geográfica. Todos estes trabalham encontram um diferencial de salários significativo associado à educação e enfatizam a necessidade de se investir nos estágios iniciais da educação, pois a taxa de retorno era bem mais elevada.

Diante dos problemas ora considerados, o presente trabalho tenta preencher uma lacuna ainda não estudada no Brasil, ao relacionar a variável educação endógena com os seguintes instrumentos: o número de escolas, população, legislação, e PIB para a variável educação. O trabalho é original ao utilizar os instrumentos acima relacionados à variável educação com a utilização de Cross-sections repetidas, ou seja, as PNADS entre os anos de 1997 e 2007.

\section{METODOLOGIA}

A equação de rendimentos visa relacionar os rendimentos auferidos por um trabalhador com suas características pessoais (educação, idade, sexo, cor) e com as características regionais. O principal objetivo associado a um procedimento que visa estimar uma equação comportamental diz respeito à questão causal ${ }^{4}$. A maneira ideal de garantir que a relação entre duas variáveis seja causal seria trabalhar com contrafactuais, ou seja, observar o trabalhador em dois momentos distintos e controlando por condicionantes salariais, inclusive o tempo.

$\mathrm{Na}$ equação de rendimentos, a forma funcional mais comum é a de Mincer (1974), no qual supõe que a questão de interesse seja estimar uma relação do logaritmo do salário $(\mathrm{lw})$ e uma variável $\mathrm{x}$, tal como: $\mathrm{lw}=\mathrm{f}(\mathrm{x})$. Cabe ressaltar que a especificação log-linear da equação de salários é pelo fato de que em muitos casos a distribuição desta variável aproxima-se bem de uma distribuição log-normal.

Assim, de acordo com o modelo de Mincer (1974), o logaritmo do rendimento individual em um dado período de tempo pode ser decomposto dentro de uma função aditiva e linear de um termo da educação (S), um termo de experiência (X) e um termo de experiência quadrática $\left(\mathrm{X}^{2}\right)^{5}$. Geralmente adota-se a forma funcional em que os ganhos se elevam à medida que os anos passam. Além disso, esses ganhos seriam crescentes a taxas decrescentes ao longo dos anos de vida do indivíduo, então, há a adoção da seguinte equação:

$$
\ln y=\alpha+\beta S+B X+B X^{2}+\varepsilon_{i t}
$$

\footnotetext{
${ }^{4}$ Ver Angrist e Krueger (1999).

${ }^{5}$ Um trabalho clássico que envolve a relação entre rendimentos e idade pode ser considerado por Murphy e Welch (1990) os quais atestam sobre a especificação quadrática da equação de rendimentos.
} 
O problema com qualquer tentativa de estimação por MQO de uma equação de rendimentos é que a variável resultante depende da heterogeneidade de cada trabalhador, que é, em grande medida, não observável. Este potencial depende de fatores como habilidade, capacidade de adaptação a mudanças, capacidade de criatividade, capacidade de relacionamento e ambição etc. É razoável admitir que o potencial de obter rendimentos é correlacionado com alguns dos principais determinantes (observáveis) dos salários, como: escolaridade, região de domicílio, sexo, e raça. Isso faz com que haja uma invalidação da interpretação causal dos coeficientes estimados.

O método mais fácil de lidar com o problema de causalidade é a inclusão de variáveis de controle observáveis que capturem a correlação entre o regressor de interesse e os componentes aleatórios. No caso do efeito da educação sobre os salários, por exemplo, vários estudos incluem resultados de testes de Quociente de inteligência ${ }^{6}$.

O problema dessa metodologia é que dificilmente podemos admitir que uma variável de controle capture toda correlação entre o regressor e o erro para auferir rendimentos. Isso só vale quando se conhece o processo que gera o regressor de interesse. Os problemas adicionais dessa metodologia são que se a variável de interesse for medida com erro, a inclusão de controles tenderá a agravar o viés atenuador decorrente desse erro. A ideia do trabalho é utilizar variações exógenas para aproximar os experimentos aleatórios.

Desse modo, seria necessária uma variável que fosse correlacionada com educação, porém não correlacionada com a capacidade de obtenção de rendimentos do trabalho, tal como: número de escolas, por exemplo. Grande parte da literatura americana trabalha com episódios esporádicos como sorteio para serviço militar ${ }^{7}$ ou mudanças nas regras que definem tamanho de classes para identificar seu efeito sobre rendimento escolar. Sendo, assim o retorno à educação adotará as seguintes hipóteses:

$$
\begin{aligned}
& \ln y=\beta_{0}+\beta_{1}+\beta_{2} x_{2}+\ldots+\beta_{K} x_{K}+u \\
& E(u)=0, \operatorname{Cov}\left(x_{j}, u\right)=0, \mathrm{j}=1,2, \ldots, \mathrm{K}-1
\end{aligned}
$$

Uma das razões para a existência de endogeneidade seria a de que anos de estudo não são sufientes para explicar o retorno à educação. Então, o método de variáveis instrumentais providencia uma solução geral para o problema de endogeneidade da variável explanatória. Pela identificação pode-se escrever a equação 2 em termos de momento populacional das variáveis observáveis. Então, tem-se:

$$
y=x \beta+u
$$

\footnotetext{
${ }^{6}$ Ver Card (1995).

${ }^{7}$ Ver Angrist (1990).
} 
Onde $x=\left(1, x_{2}, \ldots, x_{k}\right)$ e o vetor de todas as variáveis exógenas, ou seja, instrumentos correlacionados com a variável educação podem ser representado como: $z=\left(1, x_{2}, \ldots, x_{k-1}, z_{1}\right)$.

Por derradeiro, dado uma amostra aleatória $\left\{\left(x_{i}, y_{i}, \mathrm{Z}_{i 1}\right): i=1,2, \ldots, N\right\}$ para a população, o estimador de variáveis instrumentais para $\beta$ pode ser obtido como:

$$
\hat{\beta}=\left(N^{-1} \sum_{i=1}^{n} z_{i}^{\prime} x_{i}\right)^{-1}\left(N^{-1} \sum_{i=1}^{n} z_{i}^{\prime} y_{i}\right)=\left(z^{\prime} x\right)^{-1} z^{\prime} y
$$

A consistência deste estimador baseia-se na lei dos grandes números. A hipótese básica do modelo de variáveis instrumentais é que a única razão pela qual $y$ varia com $z_{i}$ é porque $x_{i}$ varia com $z_{i}$. No problema do artigo, esse estimador é equivalente ao de Mínimos Quadrados Ordinários em dois estágios, que usa os valores previstos de uma regressão de $x_{i}$ e $z_{i}$ no primeiro estágio como regressor no segundo estágio.

Então, o artigo utiliza instrumentos que atentem para o problema da endogeneidade da variável educação na equação de salários. Assim, procurou-se estabelecer a relação entre escolarização e outras variáveis com o mesmo intuito da literatura internacional, qual seja, o problema da endogeneidade educacional. Dessa maneira, adotou-se a variável número de escolas no estado e no ano de nascimento do indivíduo com o propósito de mostrar que o aumento da oferta de escolas tem efeito sobre a escolarização do aluno.

Além disso, incluímos também a variável população na lista dos instrumentos para dar uma ideia de haver uma competição por recursos educacionais entre estados, ou seja, combinando escolas e população ter-se-ia uma ideia de escolas por população e uma medida de distância do aluno em relação à escola.

Já a inclusão da variável participação do PIB estadual no PIB nacional tem como o objetivo mostrar os efeitos do mercado de trabalho na decisão educacional, ou seja, estados com maior participação no PIB teriam um mercado de trabalho mais desenvolvido, o que aumentaria os custos de oportunidade do estudo. Então, quando maior o PIB, menor deveria ser a probabilidade de o aluno permanecer na escola.

Também incluímos o impacto da Lei 5692 de 1971 (também chamada de LDB de 1971, a qual modificou a LDB de 1961). O modelo adiciona coortes de geração a qual consiste controlar o efeito das gerações sobre a educação. Nesse intuito, construiu-se também dummies de estado para todos os estados brasileiros. Como estratégia de identificação, foi feita a hipótese de considerar a aprovação escolar controlando pelo número de escolas em cada estado, participação do PIB estadual no PIB nacional, e população quando os indivíduos nascem, ou seja, como exemplo, pode-se dizer que se a data de nascimento da criança for 1948 considera-se o número de escolas em 1948 para cada Unidade de Federação (Estado). Ao obter as Pesquisas Nacionais por Amostras Domiciliares, foram considerados indivíduos com 18 anos ou mais. No primeiro estágio, será estimada a forma reduzida do modelo: 


\section{Modelo MQO (Mínimos Quadrados Ordinários)}

$$
\begin{aligned}
& \text { educa }=\alpha+\beta_{1} \text { esc }+\beta_{2} \text { pib }+\beta_{3} \text { pop }+\beta_{4} \text { Lei5692 } / 1971+\beta_{5} \text { Const } 67+\beta_{6} \text { idade }+\beta_{7} \text { idade } 2+ \\
& +\beta_{8} \text { raca }+\beta_{9} \text { sex } 1+\text { dummies de coorte }+ \text { dummies de estado }+ \text { dummies de ano }+\varepsilon_{i t}
\end{aligned}
$$

Sendo:

educa $=$ Variável dependente

$\mathrm{a}=$ Constante

esc $=$ Número de escolas anual em cada estado quando o indivíduo nasce.

pop $=$ População anual em cada estado quando o indivíduo nasce.

PIB = Participação estadual no PIB nacional anual em cada estado quando o indivíduo nasce.

Lei 5692 de 1971 = Lei de diretrizes e bases que modificou a LDB de 1961, também chamada de LDB de 1971, a qual aglutinou os quatro anos do primário foram aglutinados com os quatro anos do ginásio, ratificando o ensino de primeiro grau obrigatório de oito anos letivos.

Const 1967 = A Constituição brasileira de 1967 dispõe que o ensino dos 7 aos 14 anos seria obrigatório para todos e gratuito nos estabelecimentos primários oficiais (item II - art. 168).

raça $=$ Raça do indivíduo sendo igual a 1 quando o mesmo é branco, e 0 caso contrário.

sex1 = Sexo do indivíduo sendo igual a 1 quando o mesmo é homem, e 0 caso contrário

dummies de coorte $=1947-1950,1951-1955,1956-1960,1961-1963,1964-$ 1966, 1967-1969, 1970-1973, 1974-1978, 1979-1984, 1985-1989.

dummies de estados = RO,AC, AM, RR, AP, MA, PI, CE, RN, PE, PB, SE, AL, BA, ES, MG, RJ, SP, PR, SC, RS, MTS, GTB.

dummies de ano $=1997,1998,1999,2001,2002,2003,2004,2005,2006$, 2007.

eit $=$ Erro aleatório.

No segundo estágio, vamos estimar os retornos à educação considerando-se o impacto da variável idade, idade ao quadrado, sexo, cor e dummies de estado. Dessa forma, temos a seguinte especificação:

$$
\begin{aligned}
& l w=\alpha+\beta_{1} \text { educa }+\beta_{2} \text { idade }+\beta_{3} \text { idade }{ }^{2}+\beta_{4} \text { raca }+\beta_{5} \text { sex } 1+\text { dummies de coorte } \\
& + \text { dummies de estado }+ \text { dummies de ano }+\varepsilon_{i t}
\end{aligned}
$$

Sendo:

lw = logaritmo natural do salário

educa = educação do indivíduo (estimada considerando os instrumentos: número de escolas quando o indivíduo nasce, população quando o indivíduo nasce, participação regional do produto interno bruto quando o indivíduo nasce, e a Lei 5692 de 1971). 
idade $=$ número de anos do indivíduo.

idade $^{2}=$ idade ao quadrado capta retornos a experiência.

raça $=1$ se o indivíduo for branco, 0 caso contrário.

sex1 = variável que admite 1 se o sexo for masculino, 0 caso contrário.

dummies de estado = RO,AC, AM, RR, AP, MA, PI, CE, RN, PE, PB, SE, AL, BA, ES, MG, RJ, SP, PR, SC, RS, MTS, GTB.

dummies de coorte $=1947-1950,1951-1955,1956-1960,1961-1963,1964-$ 1966, 1967-1969, 1970-1973, 1974-1978, 1979-1984, 1985-1989.

dummies de ano $=1997,1998,1999,2001,2002,2003,2004,2005,2006$, 2007.

eit $=$ erro aleatório

\section{DESCRIÇÃO DOS DADOS}

A análise empírica será baseada nas PNADS de 1997 até 2007 que é publicada anualmente pelo IBGE. Consideramos as variáveis retiradas das estatísticas do século XX no sítio do IBGE www.ibge.gov.br: número de escolas, e população no estado e no ano de nascimento do indivíduo como instrumentos para educação. Os salários foram deflacionados de acordo com a Tabela 1.

Além disso, consideramos participação estadual do PIB regional no PIB nacional que também é uma base de dados do IBGE. Cabe destacar que os salários (renda) foram deflacionados a partir de dados do INPC do IBGE com base na metodologia de Corseuil e Foguel (2002). A Tabela 1 mostra os valores para deflacionar os salários das PNADS considerando como base o ano de 2007. Ante o fato de se ter apenas obtido dados de participação estadual no PIB nacional a partir de 1947, o presente estudo inicia-se em 1947.

Houve a agregação de Goiás, Tocantins, e Brasília como forma de compatibilizar os dados, dado que haveria problema de viés ao omitir pessoas nascidas antes da cisão de Tocantis e da criação de Brasília. Houve também a agregação do Mato Grosso com Mato Grosso do Sul, dado que ao considerar a cisão perderíamos observações de indivíduos que nasceram antes da separação dos estados, então, teríamos viés amostral.

Tabela 1: Valores para Deflacionar Rendas

\begin{tabular}{ccc}
\hline Ano & Semana de referência & Deflator \\
\hline 1997 & $21 / 09$ a $27 / 09$ & 36.08 \\
1998 & $20 / 09$ a $26 / 09$ & 39.14 \\
1999 & $19 / 09$ a $25 / 09$ & 44.16 \\
2001 & $23 / 09$ a $29 / 09$ & 57.09 \\
2002 & $20 / 09$ a $26 / 09$ & 66.33 \\
\hline
\end{tabular}




\begin{tabular}{ccc}
\hline 2003 & $21 / 09$ a $27 / 09$ & 82.43 \\
2004 & $19 / 09$ a $25 / 09$ & 88.37 \\
2005 & $18 / 09$ a $24 / 09$ & 92.95 \\
2006 & $24 / 09$ a $30 / 09$ & 95.35 \\
2007 & $23 / 09$ a $29 / 09$ & 100.00 \\
\hline
\end{tabular}

Dados gerados pelos autores.

\section{RESULTADOS}

Para a construção do banco de dados e estimação das regressões, o trabalho considera a data de nascimento de cada indivíduo em cada PNAD em cada uma das gerações apresentadas nas equações anteriores. Então, temos indivíduos que apresentam 18 anos ou mais em 2010. Dessa maneira, os indivíduos da amostram apresentam uma maior probabilidade de ter completado e/ou estar completado o ciclo educacional no estado de nascimento e de acordo com geração a qual nasceu. A utilização de coortes é uma prática muito comum na literatura de retorno à educação, e, destaca-se entre os trabalhos, o artigo de Ichino, Winter, e Ebmer (1998), o qual foi mencionado anteriormente.

A Tabela 2 mostra as estatísticas descritivas das variáveis para a 1 considerando a PNAD de 1997 até 2007, logo, nota-se que mais que $92 \%$ da amostra apresenta mais do que 1 ano de educação, $81,87 \%$ da amostra tem mais que quatro anos de educação e aproximadamente $55 \%$ tem mais do que oito anos de educação. É possível notar que na amostra que os indivíduos têm em média mais do que sete anos de escolaridade. Então, a variável educa mostra que a média educacional da amostra é de aproximadamente oito anos de estudo.

Tabela 2: Estatísticas descritivas para a região Sul utilizando as PNADS de 1997 a 2007

\begin{tabular}{lccccc}
\hline & Observações & Média & Desvio-Padrão & Mínimo & Máximo \\
Escolariza $\geq 1$ & 1248998 & .9237 & .2654 & 0 & 1 \\
Escolariza $\geq 4$ & 1248998 & .8187 & .3853 & 0 & 1 \\
Escolariza $\geq 8$ & 1248998 & .5496 & .4975 & 0 & 1 \\
Idade & 1248998 & 35 & 10 & 18 & 60 \\
Sex1 (Masculino) & 1248998 & .5965 & .4905 & 0 & 1 \\
raca2 (Branco) & 1248998 & .5055 & .5000 & 0 & 1 \\
Educa & 1248998 & 7.7126 & 4.3456 & 0 & 15 \\
Data nascimento & 1248998 & 1967.916 & 10.40 & 1947 & 1989 \\
Esc & 1248998 & 10034 & 6734 & 13 & 26191 \\
PIB & 1248998 & .0927 & .1186 & 0 & .4273929 \\
\hline
\end{tabular}




\begin{tabular}{lccccc}
\hline Pop & 1248998 & 6850002 & 5595552 & 14000 & $3.09 \mathrm{e}+07$ \\
Constituição 67 & 1248998 & .6160 & .4863 & 0 & 1 \\
Lei 5692 de 1971 & 1248998 & .4341 & .4956 & 0 & 1 \\
\hline
\end{tabular}

Fonte: Tabela elaborada pelos autores.

Então, já que foram mencionadas as estatísticas descritivas, podemos prosseguir no estudo e estimar os modelos econométricos. Na Tabela 3 infere-se a relação entre educação e controles de forma a mostrar o primeiro estágio da regressão, ou seja, Educação contra Soma de escolas, população, participação no PIB, leis que mudaram faixa etária de escolarização, regras que mudaram a probabilidade de escolarização, e dummies de coorte, dummies de estado, sexo, raça etc.

Logo, conforme apresentado na metodologia, temos quatro tipos de modelo ponderados pela participação do peso do indivíduo na PNAD: i) Modelo que considera apenas o efeito do número de escolas, PIB, e população sobre a probabilidade de escolarização; ii) Modelo que adiciona o efeito da Constituição de 1967 sobre a escolarização dos indivíduos; iii) Modelo que adiciona o efeito da Lei 5692 de 1971 sobre a escolarização dos indivíduos; v) modelo que não considera o impacto da variável população, e admite o impacto da Lei 5692 de 1971 sobre a probabilidade de escolarização, número de escolas, e PIB.

A Tabela 3 mostra as estimações dos modelos de Mínimos Quadrados Ordinários que utilizam como controles: o número de escolas, a participação do PIB estadual, população, Constituição de 1967, e Lei 5692 de 1971. Abaixo de cada um dos modelos estimados foram considerados os erros-padrão entre parêntese com o respectivo nível de significância estatística. Neste subitem, há uma junção das PNADS de 1997, 1998, 2000, 2001, 2002, 2003, 2004, 2005, 2006 e 2007.

Houve a tentativa de modelar as variáveis: sexo, educação, raça, idade, idade ao quadrado, dummies de ano e dummies de coorte. A amostra irá considerar indivíduos com mais de 18 anos. Não foi estimado um painel pelo fato de não ser a mesma população ao longo do tempo e pelo fato de haver problema de identificação na estimação de um painel que considere sexo, raça e educação. As dummies de estado consideram o estado de São Paulo como dummy de comparação.

As dummies de coortes controlam pelo impacto da aprovação escolar quando o indivíduo nasce. Nesta parte do trabalho foram utilizados, além das PNADS acima referidas, dados do IBGE referentes a número de escolas, população e participação estadual no PIB, e dummies de Legislação, ou seja, o efeito da Constituição de 1967 após o ano de 1967, e o efeito da Lei 5692 de 1971 após o ano de 1971.

Ao identificar o modelo, considerou-se o impacto do sexo masculino (sex1) na aprovação escolar em relação ao sexo feminino e o impacto da raça branca (raca2) sobre as demais raças. Ainda na Tabela 3, observa-se no modelo 1: o número de escolas e o PIB estadual no ano e na data de nascimento do indivíduo apresentarem um efeito positivo sobre a probabilidade de escolarização. Já a variável população apresenta um resultado próximo a zero sobre a probabilidade de escolarização. Nas demais variáveis temos um resultado que já foi comprovado pela literatura, ou 
seja, idade tem um efeito positivo sobre a educação, mas a taxas decrescentes, homens (sex1) apresentam uma maior probabilidade de escolarização, e indivíduos brancos apresentam uma maior probabilidade de escolarização.

O modelo 2 da Tabela 3 considera as mesma variáveis do modelo 1, e, apresenta as mesmas relações citadas acima, entretanto adiciona o efeito da Constituição de 1967 sobre a probabilidade de escolarização dos indivíduos. E, o resultado é interessante, pois a Constituição de 1967 é negativamente correlacionada com a educação dos indivíduos.

Tabela 3: Primeiro Estágio: Determinantes dos Anos de Estudo no Brasil

\begin{tabular}{|c|c|c|c|c|}
\hline & (1) & (2) & (3) & (4) \\
\hline \multirow[t]{2}{*}{ Esc } & $3.210 \mathrm{e}-05$ & $3.140 \mathrm{e}-05$ & $3.20 \mathrm{e}-05$ & $2.88 e-05$ \\
\hline & $(2.28 \mathrm{e}-06)^{* * *}$ & $(2.29 \mathrm{e}-06)^{* * *}$ & $(2.28 \mathrm{e}-06)^{* * *}$ & $(2.17 \mathrm{e}-06)^{* * *}$ \\
\hline \multirow[t]{2}{*}{ PIB } & 4.199 & 4.529 & 4.171 & 3.292 \\
\hline & $(.360)^{* * *}$ & $(.395)^{* * *}$ & $(.360)^{* * *}$ & $(.365)^{* * *}$ \\
\hline \multirow[t]{2}{*}{ Pop } & $-9.01 e-09$ & $-2.42 e-09$ & $-8.91 e-09$ & Não \\
\hline & $(2.15 e-09)^{* * *}$ & $(4.00 e-09)$ & $(2.15 \mathrm{e}-09)^{* * *}$ & \\
\hline \multirow[t]{2}{*}{ Lei 5682 de 1971} & Não & Não & .063 & .065 \\
\hline & - & - & $(.027)^{* * *}$ & $(.027)^{* *}$ \\
\hline \multirow[t]{2}{*}{ Const67 } & Não & -.081 & Não & Não \\
\hline & - & $(.030)^{* * *}$ & & \\
\hline \multirow[t]{2}{*}{ Idade } & .133 & .133 & .133 & .138 \\
\hline & $(.005)^{* * *}$ & $(.005)^{* * *}$ & $(.005)^{* * *}$ & $(.005)^{* * *}$ \\
\hline \multirow[t]{2}{*}{ Idade2 } & -.003 & -.003 & -.003 & -.003 \\
\hline & $(6.50 e-05)^{* * *}$ & $(6.50 e-05)^{* * *}$ & $(6.50 e-05)^{* * *}$ & $(6.50 \mathrm{e}-05)^{* * *}$ \\
\hline \multirow[t]{2}{*}{ Sex1 } & -1.302 & -1.301 & -1.302 & -1.302 \\
\hline & $(.008)^{* * *}$ & $(.008)^{* * *}$ & $(.008)^{* * *}$ & $(.008)^{* * *}$ \\
\hline \multirow[t]{2}{*}{ Raca2 } & 1.850 & 1.850 & 1.850 & 1.850 \\
\hline & $(.009)^{* * *}$ & $(.009)^{* * *}$ & $(.009)^{* * *}$ & $(.009)^{* * *}$ \\
\hline Dummies de Coorte & Sim & Sim & Sim & Sim \\
\hline Dummies de Estado & Sim & Sim & Sim & Sim \\
\hline Dummies de Ano & Sim & Sim & Sim & Sim \\
\hline \multirow[t]{2}{*}{ Const. } & 4.497 & 4.439 & 4.420 & 4.364 \\
\hline & $(.153)^{* * *}$ & $(.173)^{* * *}$ & $(.156)^{* * *}$ & $(.153)^{* * *}$ \\
\hline Observações & 1248998 & 1248998 & 1248998 & 1248998 \\
\hline Teste F & 4483.77 & 4304.71 & 4392.39 & 4480.08 \\
\hline Prob $>$ F & 0.0000 & 0.0000 & 0.0000 & 0.0000 \\
\hline $\mathrm{R} 2$ & 0.1543 & 0.1543 & 0.1543 & 0.1543 \\
\hline Root MSE & 4.0116 & 4.0116 & 4.0116 & 4.0116 \\
\hline
\end{tabular}

*** significante a $1 \% ;{ }^{*}$ significante a $5 \%$; ${ }^{*}$ significante a $10 \%$

É relevante salientar que a elaboração da nova Constituição brasileira, que se deu em janeiro de 1967, e no tema educação, o segundo item do artigo 168 aumen- 
tou a faixa de escolarização que era de 7 a 11 anos para 7 aos 14 . No entanto, não havia uma relação entre idade e anos de educação, no sentido de haver a preocupação do indivíduo estar matriculado na série correta de acordo com a idade correta. Então, talvez pela não articulação real do primeiro ciclo do ensino médio com o primário é que observamos o resultado negativo da constituição de 1967 que já foi e constatado no modelo 2 da Tabela 3.

Cabe agora ao trabalho testar se a Lei 5692 de 1971 teve efeito sobre a escolarização dos indivíduos ao longo das PNADS analisadas no trabalho. Então, o modelo 3 incorpora o efeito da Lei 5692 de 1971 e não incorpora o efeito da Constituição de 1967, pelos resultados da variável Lei 5692 de 1971 está correlacionada positivamente com educação.

A elaboração da Lei ${ }^{\circ}$ 5.692/71 promoveu a integração vertical dos graus, níveis de ensino, das atividades, área de estudo e disciplinas, bem como instituiu a integração horizontal dos ramos de ensino e das áreas de estudo e disciplinas entre si, contribuiu positivamente para a educação brasileira.

Então, após as observações supracitadas, constata-se no modelo 4 da Tabela 3 que o efeito da Lei 5692 de 1971 sobre a probabilidade de escolarização é mais significativo quando não consideramos o impacto da variável população, confirmando mais uma vez o efeito positivo da Lei 5692 de 1971 sobre a variável educação. Então, a partir dos resultados encontrados na Tabela 3, considerou-se como um exercício adicional o impacto da Lei 5692 de 1971 sobre o segundo estágio da regressão.

Constata-se também, segundo as estimações apresentadas na Tabela 3, o impacto praticamente nulo da variável população na variável educação. Então, será feito um segundo estágio da regressão que não considera a variável população como instrumento e constará no modelo 4 da Tabela 4. Então, o modelo 4 da tabela 4 irá considerar como instrumentos: a soma do número de escolas no estado e na data de nascimento do indivíduo, o PIB no estado e na data de nascimento do indivíduo, e a Lei 5692 de 1971.

Ao se estimar as equações de rendimento convencional e com os instrumentos número de escolas, a população, PIB, e leis que afetam escolaridade para a variável educação, foram obtidos quatro modelos na Tabela 4 com seus respectivos resultados: i) $\mathrm{O}$ primeiro modelo considera a equação de salários que não instrumentaliza por número de escolas, população e PIB; ii) O segundo modelo é estimado por variáveis instrumentais e considera os instrumentos número de escolas, população, PIB, as dummies de coorte, e o peso do indivíduo na PNAD nos dois estágios; iii) $\mathrm{O}$ terceiro modelo considera todas as especificações do segundo modelo e adiciona o impacto da lei 5692 de 1971; iv) O quarto modelo considera todas as especificações do terceiro modelo, mas não considera variável população como instrumento.

Assim, será estimada uma equação de rendimentos convencional no modelo 1 da Tabela 4 que considerará as PNADS de 1997 a 2007 e será ponderada pelo peso do indivíduo na amostra. Após isso, procurará instrumentalizar a educação pelo número de escolas por estado, população por estado e PIB estadual no ano de nascimento do indivíduo. Logo, há a preocupação de trazer à tona o problema de 
endogeneidade e utilizar instrumentos, tendo como consequência uma equação de segundo estágio.

De acordo com a Tabela 4, observou-se no primeiro modelo que um ano de escola contribui em mais de $11 \%$ do rendimento do indivíduo. No que se refere ao sexo pode-se afirmar, segundo os modelos estimados, que homens ganhariam mais do que mulheres todos os modelos estimados. No que se refere ao efeito de raça, pode-se afirmar que brancos ganham mais que não brancos em todos os modelos, entretanto com a utilização de instrumentos o impacto positivo da raça branco é mais evidente sobre os salários.

Os resultados interessantes dos modelos 2, 3, 4 da Tabela 4 corroboram que há uma atenuação do impacto da educação sobre os salários, deste modo, ao se instrumentalizar a educação tem-se resultados menores para cada ano de educação em relação ao modelo que considera apenas o primeiro estágio, e, estes resultados estão bem próximos dos resultados de Duflo (2001), quando foi observado o impacto do número de escolas sobre a probabilidade de escolarização.

O modelo 4 da Tabela 4, que é o objetivo do artigo, pode-se constatar que o retorno a educação é ainda mais atenuado quando consideramos apenas os instrumentos: soma do número de escolas no ano e na data de nascimento do indivíduo, participação do PIB estadual no PIB nacional no ano e na data de nascimento do indivíduo, e da Lei 5692 de 1971.

Tabela 4: Segundo Estágio: Equações de rendimento

\begin{tabular}{ccccc}
\hline Modelo & $\mathrm{I}$ & $\mathrm{II}$ & $\mathrm{III}$ & $\mathrm{IV}$ \\
\hline Educa & .116 & .088 & .094 & .055 \\
& $(.183 \mathrm{e}-04)^{* * *}$ & $(.008)^{* * *}$ & $(.008)^{* * *}$ & $(.008)^{* * *}$ \\
Idade & .090 & .093 & .093 & .098 \\
& $(.935 \mathrm{e}-04)^{* * *}$ & $(.001)^{* * *}$ & $(.001)^{* * *}$ & $(.002)^{* * *}$ \\
idade2 & $-.876 \mathrm{e}-03$ & $-.958 \mathrm{e}-03$ & $-9.42 \mathrm{E}-04$ & -.001 \\
& $(.166 \mathrm{e}-04)^{* * *}$ & $(.270 \mathrm{e}-04)^{* * *}$ & $(2.67 \mathrm{E}-05)^{* * *}$ & $(2.90 \mathrm{e}-05)^{* * *}$ \\
sex1 & .526 & .490 & .497 & .447 \\
Raça & $(.002)^{* * *}$ & $(.011)^{* * *}$ & $(.011)^{* * *}$ & $(.012)^{* * *}$ \\
& .136 & .188 & .177 & .177 \\
Dummies de Coorte & $(.001)^{* * *}$ & $(.015)^{* * *}$ & $(.015)^{* * *}$ & $(.015)^{* * *}$ \\
Dummies de Estado & $\mathrm{Sim}$ & $\mathrm{Sim}$ & $\mathrm{Sim}$ & $\mathrm{Sim}$ \\
Dummies de Ano & $\mathrm{Sim}$ & $\mathrm{Sim}$ & $\mathrm{Sim}$ & $\mathrm{Sim}$ \\
Constante & $\mathrm{Sim}$ & $\mathrm{Sim}$ & $\mathrm{Sim}$ & $\mathrm{Sim}$ \\
& 2.953 & 3.000 & -.622 & -.621 \\
& $(.013)^{* * *}$ & $(.056)^{* * *}$ & $(.056)^{* * *}$ & $(.056)^{* * *}$ \\
\hline
\end{tabular}

*** significante a $1 \%$; ${ }^{*}$ significante a $5 \%$; ${ }^{*}$ significante a $10 \%$

\section{CONCLUSÕES}

A Lei 5692 de 1971, onde os quatro anos do primário foram aglutinados com os quatro anos do ginásio, ratificando o ensino de primeiro grau obrigatório de 
oito anos letivos. E este trabalho prova que a Lei 5692 de 1971 teve um impacto positivo sobre a probabilidade de escolarização. O trabalho demonstra a importância das variáveis: número de escolas, PIB estadual, e Lei 5692 de 1971 para a escolarização da população brasileira.

O artigo também apresentou que número de escolas é uma variável importante para a escolarização das pessoas. No mais, os estados com maior PIB apresentam uma maior probabilidade de escolarização. O trabalho mostrando um problema de discriminação no mercado de trabalho, pois homens brancos teriam um maior retorno a educação do que mulheres.

No que se refere à idade, pode-se afirmar que ao longo do ciclo de vida, à medida que as pessoas envelhecem, há uma menor probabilidade de escolarização. A escolha dos instrumentos: número de escolas, população, e participação do PIB estadual no PIB regional, Lei 5692 de 1971 advieram do fato dos mesmos ser exógenos. Não se utilizou painel pelo fato de controlar por características observáveis, pois controlando por efeito fixo poder-se ia não estar encontrando o resultado correto pelo fato de não se estar trabalhando com os mesmos indivíduos ao longo do tempo.

Por fim, a relação entre educação é salários é atenuada quando se considera o método por variáveis instrumentais, ou seja, apesar da Lei 5692, do PIB, e, do Número de Escolas ter impacto positivo sobre a probabilidade de escolarização, o retorno à educação acaba por ser reduzido ficando em torno do valor estimado pela literatura. Dessa feita, a educação tem impacto significativo sobre os salários, mas bem menores quando são consideras: a Lei 5692 de 1971, a participação do PIB estadual no PIB nacional, e a soma do número de escolas em cada estado e ano quando o indivíduo nasce. Mesmo assim, podemos dizer que a Lei 5692 de 1971 impacta positivamente sobre a educação e modificou significativamente a educação brasileira.

\section{REFERÊNCIAS BIBLIOGRÁFICAS}

ANGRIST, J. (1990) "Lifetime earning and the Vietnam era draft lottery: evidence from social security administrative records" American Economic Review, v. 80, no 3, p. 313-335.

ANGRIST, J. and KRUEGER, A. (1999) “Empirical strategies in labor economics”. In: Ashenfelter, O \& Card, D. Handbook of Labor Economics, v.3A, Elsevier. p.1277-1366.

ANGRIST, J. and KRUEGER, A. (1991) "Does compulsory school attendance affect schooling and earnings?” June 1990, Quarterly Journal of Economics Vol. 106, No. 4, November,p.979-1014

ASHENFELTER, O. et al. (1999) "A review of estimates of the schooling/earnings relationship, with tests for publication bias", Labour Economics 6 (4), p.453-470.

BARROS, R. e RAMOS, L. (1994) "A note on the temporal evolution of the relationship between wages and education among Brazilian primeage males - 1976/89”, In: Mendonça, R, Urani, A. Estudos Sociais e do Trabalho. Rio de Janeiro: IPEA.p. 31-53.

BECKER, G. (1964) Human Capital. New York: NBER, p. 62.

BEHRMAN, J. and BIRDSALL, N. (1983) “The quality of schooling: quantity alone is misleading”. American Economic Review, v. 73, p. 928-946. 
BRASIL (2010) Lei número 4.024 de 20 de dezembro de 1961, disponível em: < http:// www.senado. gov.br/sf/legislacao/BasesHist > acesso em 2010.

BRASIL (2012) Lei número 5.692 de 1971, disponível em: <http:// www.senado.gov.br/sf/legislacao/ BasesHist > acesso em 2010.

CARD, D. (1995) "Earnings, schooling, and ability revisited" Research in Labor Economics, vol. 14, Greenwich Connecticut: JAI Press, p. 23-48.

CORSEUIL, C.; FOGUEL, M. (2002) Uma sugestão de deflatores para rendas obtidas a partir de algumas pesquisas domiciliares do IBGE” Texto para Discussão 897, julho de 2002. p.1-8.

CORSEUIL, A. et al. (2002) "Fatores que determinam o nível salarial no setor formal brasileiro", In: Corseuil et al. Estrutura Salarial: Aspectos Conceituais e Novos Resultados para o Brasil. IPEA. p. 101-143.

DUFLO, E. (2001) Schooling and labor market consequences of school construction in Indonesia: Evidence form an unusual policy experiment", American Economic Review. Vol 91, No 4, 795-813.

FERNANDES, R. (2002) “Desigualdade salarial: Aspectos teóricos”. In: Corseuil et al. Estrutura Salarial: Aspectos Conceituais e Novos Resultados para o Brasil. IPEA. p. 1-50.

GRILICHES, Z. (1977) "Estimating the returns to schooling: some econometric problems", Econometrica. Vol. 45. Number 1. January. 1-22.

HARMON, C. and WALKER, I. (1995) "Estimates of the economic return to schooling for the United Kingdom”, American Economic Review, Vol. 85, p. 1278-1286.

HASENBALG. (2002) "Estatísticas do século XX: Educação. In.: IBGE. Estatísticas do século XX, disponível em http:॥ www.ibge.gov.br. Acesso em agosto de 2004.

ICHINO, A and WINTER-EBMER, R (1998) "The long run education cost of world war II. An example of local average treatment effect estimation". EUI, 10, p.1-30.

KANE, T. and ROUSE, C. (1993) Labor market returns to two-and for-year college: is a credit really a credit emhancy do degrees maters? Cambridge, MA, and Princenton, NJ: Havard University and Princenton University. Working Paper no 4268.

KASSOUF, A (1998) "Wage gender discrimination and segmentation in the Brasilian labor market", Economia Aplicada., v.2., p. 243-269.

LANGONI, C. (1973) Distribuição de renda e desenvolvimento econômico do Brasil. Rio de Janeiro: Editora Expressão e Cultura, p. 315.

LEAL, C e WERLANG, S. (1991) “Retornos à educação no Brasil: 1976/89”. Pesquisa e Planejamento Econômico, Rio de Janeiro, v. 21, n.3, p. 423-448.

LIGHT, A. (2001) In-school work experience and the returns tos schooling", Journal of Labor Economics, Vol. 19. no 1. 65-92.

LOURY, L and GARMAN, D. (1995) “College selectivity and earnings”, Journal of Labor Economics. Vol.13. no 2. p. 289-308.

MENEZES-FILHO, N. (2002) "Equações de Rendimentos: Questões Metodológicas”. In: Corseuil et al. (2002) Estrutura Salarial: Aspectos Conceituais e Novos Resultados para o Brasil. IPEA. p. 51-66.

MINCER, J. (1974) Schooling, experience e earnings. Columbia University Press.

MURPHY, K and WELCH, F. (1990) “Empirical age-earning profiles" Journal of Labor Economics, Vol 8. no 2. p. 202 -229.

RAMOS, L. (1991) "Educação, desigualdade de renda e ciclo econômico no Brasil”, Pesquisa e Planejamento Econômico, Rio de Janeiro. v.21, n.3, p.559-574.

SENNA, J. (1976) "Escolaridade, experiência no trabalho e salários no Brasil”, Revista Brasileira de Economia, v. 30, n.2. p. 163-193.

TANNEN, M. (1991) “New estimates of the returns to schooling in Brazil”, Economics of Education Review. v 10, n. 2. p. 123-135,

WELCH, F. (1975) "Human capital theory: education, discrimination, and life cycles. The human capital approuch: an appraisal”, American Economic Review. May. p. 63-73. 